\title{
L'ANTIQUITÉ POUR CONVAINCRE. L'HISTOIRE ROMAINE DANS LES LIBELLES DE LA LIGUE PARISIENNE (1585-1594) : RHÉTORIQUE ET CIRCULATION DU SAVOIR HISTORIQUE
}

\author{
Alexandre Goderniaux
}

Presses universitaires du Midi | «Littératures classiques »

2020/1 N 101 | pages 95 à 106

ISSN 0992-5279

ISBN 9782810706945

Article disponible en ligne à l'adresse :

https://www.cairn.info/revue-litteratures-classiques-2020-1-page-95.htm

Distribution électronique Cairn.info pour Presses universitaires du Midi.

(C) Presses universitaires du Midi. Tous droits réservés pour tous pays.

La reproduction ou représentation de cet article, notamment par photocopie, n'est autorisée que dans les limites des conditions générales d'utilisation du site ou, le cas échéant, des conditions générales de la licence souscrite par votre établissement. Toute autre reproduction ou représentation, en tout ou partie, sous quelque forme et de quelque manière que ce soit, est interdite sauf accord préalable et écrit de l'éditeur, en dehors des cas prévus par la législation en vigueur en France. Il est précisé que son stockage dans une base de données est également interdit. 


\section{L'Antiquité pour convaincre L'histoire romaine dans les libelles de la Ligue parisienne $(1585-1594)$ : rhétorique et circulation du savoir historique}

L'auteur de l'Histoire admirable a la posterite des faits et gestes de Henry de Valois, libelle $^{1}$ diffusé au cours du second semestre de l'année 1589 par l'imprimeur parisien Pierre Des Hayes, affirme, péremptoire :

entre les autres profits que l'histoire nous apporte, celuy est à mon jugement le plus grand qu'elle nous met devant les yeux : les vertus de nos predecesseurs, afin que nous les imitions, et aussi elle nous propose les vices de nos devanciers, afin que nous les evitions, quoy faysans il est impossible que nous ne reglions bien et heureusement le cours de nostre vie. ${ }^{2}$

Le passé est doté d'une fonction essentiellement édificatrice : l'histoire est mâ̂tresse de vie et de prudence ; celui qui la connaît et l'interprète avec acuité ne peut que mener une vie vertueuse. Par conséquent, les 870 textes $^{3}$ publiés par la Ligue parisienne ${ }^{4}$ de 1585 à 1594 convoquent et revendications et, dans

1 Sur ce terme, voir T. Debbagi Baranova, À coups de libelles. Une culture politique au temps des guerres de Religion (1562-1598), Genève, Droz, 2012, p. 35-36 ; M. Bernard, "Vox populi vox Dei est. Procédés de la diffamation dans les libelles ligueurs du début de l'année 1589 », Albineana: Cahiers d'Aubigné, n 23/1, 2011, p. 245-266.

2 Histoire admirable a la posterite des faits et gestes de Henry de Valois. Comparez en tous poincts avec ceux de Loys Faineant : et la miserable fin de l'un et de l'autre. Avec vn nouueau et fatal Anagramme du nom dudict Henry de Valois, Paris, P. Des Hayes, 1589, p. 3-4. La graphie d'époque a été retranscrite à l'identique, à l'exception des tildes abrégeant un $m$ ou un $n$, qui ont été résolus. Nous avons également opté pour la dissimilation de $i$ et de $j$, de $u$ et de $v$.

3 D. Pallier, Recherches sur l'imprimerie à Paris pendant la Ligue (1585-1594), Genève, Droz, 1975.

4 Sur ce vaste mouvement religieux, politique et social né de la contestation des prétentions du huguenot Henri de Bourbon au trône de France à la suite de l'extinction des Valois, voir 
l'objectif presque exclusif de convaincre, d'innombrables exemples et contreexemples issus du passé. Si les polémistes se réfèrent à de multiples registres historiques, dans le domaine de l'Antiquité, c'est l'histoire romaine qui jouit de la plus grande faveur.

Dans la continuité de récentes approches ${ }^{5}$, il s'agira d'observer comment l'Antiquité romaine est concrètement convoquée par les libellistes à des fins argumentatives. Faisant des libelles son objet principal, l'étudiant avec précision avant d'envisager la problématique sous un angle plus vaste, l'enquête commencera par l'exposé d'éléments d'interprétation des pratiques libellistes et s'achèvera par l'intégration de ces données dans un plus vaste schéma de médiations. Les processus rhétoriques grâce auxquels les polémistes intègrent les éléments de l'histoire antique aux libelles seront ainsi mis au jour. Une étude de leurs sources permettra ensuite de dévoiler les médiations à l'origine de leur écriture.

\section{Rhétorique : l'histoire romaine, un répertoire d'exempla comme un autre?}

L'attribution d'une vertu édificatrice au passé et son utilisation à des fins argumentatives inscrivent la médiation ligueuse de l'histoire antique dans la plus pure tradition de l'art oratoire ${ }^{6}$. En rhétorique, «l'histoire pour l'histoire n'existe $\operatorname{pas}^{7} »$ : le passé est simplifié autant que faire se peut afin de faciliter l'extraction d'exempla, le genre de l'exemplum étant défini par Roger Chartier comme un "récit donné pour véridique, chargé d'incarner dans un ensemble particulier la vérité universelle de la proposition qu'il illustre ${ }^{8}$ ». Les formes prises par cette convocation d'exempla romains dans les libelles catholiques sont de très riches indices de la médiation ligueuse de l'histoire antique.

\section{De l'anecdote à la figure}

L'exemplum canonique est une petite histoire édificatrice narrée par l'auteur, comme dans la Declaration de Messieurs les habitans de la ville de Thoulouse:

J.-M. Constant, La Ligue, Paris, Fayard, 1996 ; N. Le Roux, Un régicide au nom de Dieu. L'assassinat d'Henri III (1 ${ }^{\text {er }}$ août 1589), Paris, Gallimard, 2006.

5 L. Avezou, «Du Moyen Âge à la fin des lumières », dans Chr. Amalvi (dir.), Les Lieux de l'histoire, Paris, A. Colin, 2005, p. 13-63 ; Chr. Jacob (dir.), Lieux de savoir. 1: Espaces et communautés, Paris, A. Michel, 2007 ; S. Van Damme (dir.), Histoire des sciences et des savoirs. 1 : De la Renaissance aux Lumières, Paris, Éd. du Seuil, 2015.

6 M. Kempshall, Rhetoric and the Writing of History (400-1500), Manchester, Manchester University Press, 2011, p. 275-284 ; P. Courroux, L'Écriture de l'histoire dans les chroniques françaises (XII $-X V^{e}$ siècle), Paris, Classiques Garnier, 2016, p. 101-110.

$7 \quad$ Ibid., p. 586.

8 R. Chartier «La pendue miraculeusement sauvée. Étude d'un occasionnel », dans R. Chartier (dir.), Les Usages de l'imprimé (XVe-XIXe siècle), Paris, Fayard, 1987, p. 92. 
Autant il en advint à Silla malheureux tyran, qui pour ce qu'il tiroit beaucoup d'argent du peuple, en tyrannisant se disoit le plus heureux que la terre porta jamais : toutesfois quelle fin eust il ? il fut mangé de vers. ${ }^{9}$

L'exemplum se rapproche ici du petit fait vrai, aussi connu sous le nom d'anecdote, qui, sous des apparences divertissantes, dissimule une stratégie rhétorique forte ${ }^{10}$. Pares cum paribus facillime una congregantur ${ }^{11}$ : chacun s'assemble très facilement avec ses égaux. Inscrit dans cette perspective analogique, l'exemplum fonctionne plus par abduction que par induction ${ }^{12}$ : un cas particulier est comparé à un autre, sans généralisation. La Declaration poursuit en effet :

Or je croy que Henry de Valois vouloit ressembler a Silla, quant il fit massacrer à Bloys Messeigneurs les Duc, et Cardinal de Guyse [...]: car Silla ne sçachant comment eviter le peril d'estre empesché de faire tyrannye, et voyant qu'il pouvoit estre contrainct, par des Seigneurs aussi grans que luy, de devenir bon Roy, il s'advisa de les faire tuer et massacrer $[\ldots] .{ }^{13}$

«Le plus souvent, l'avenir ressemble au passé ${ }^{14} »$. Selon cette conception aristotélicienne de l'histoire qui conserve toute son autorité durant la première Modernité15, l'exemplum permet, outre la compréhension du présent, l'anticipation du futur. Ainsi, puisque les mêmes causes reproduiront les mêmes effets, si les Français prient suffisamment Dieu, l'histoire se répétera totalement et Henri III connaîtra le même sort que les tyrans romains : "Je croy fermement que Henry de Valois sera puny d'une plus miserable mort que ne fut pas celle de Silla, et se verra dans peu de temps ${ }^{16}$. »

9 Declaration de Messieurs les habitans de la ville de Thoulouse auec l'arrest du Parlement de ladicte ville, Paris, M. Ioüin, 1589, p. 5. La mort comme châtiment divin logique après une vie de péchés est un élément typique des recueils de vies d'hommes illustres : voir infra et P. Eichel-Lojkine, Le Siècle des grands hommes. Les recueils de vies d'hommes illustres avec portraits du XVIe siècle, Louvain, Peeters, 2001, p. 195-203.

10 K. Abiven, L'Anecdote ou la fabrique du petit fait vrai : de Tallemant des Réaux à Voltaire (1650-1750), Paris, Classiques Garnier, 2015.

11 N. Le Fèvre, La Louange de la Ligue des seigneurs de Costentin, Paris, D. Millot, 1589, p. 10.

12 K. Abiven, "L'exemplum : un modèle opératoire dans la lettre familière ? », Exercices de rhétorique, $\mathrm{n}^{\circ}$ 6, 2016, p. 4 ; G. Declercq, L'Art d'argumenter. Structures rhétoriques et littéraires, Paris, Éditions Universitaires, 1992, p. 112-113.

13 Declaration de Messieurs les habitans de la ville de Thoulouse, op. cit., p. 5-6.

14 Aristote, Rhétorique, II, 20, 1394a, éd. A. Wartelle et M. Dufour, Paris, Les Belles Lettres, 1973, t. II, p. 106 ; cité par K. Abiven, L'Anecdote ou la fabrique du petit vrai, op. cit., p. 109.

15 B. Guion, "L'histoire maîtresse de prudence », dans É. Berriot-Salvadore, C. Pascal, Fr. Roudaut et Tr. Tran (dir.), La Vertu de prudence entre Moyen Age et âgeclassique, Paris, Classiques Garnier, 2012, p. 461-486 ; K. Pomian, L'Ordre du temps, Paris, Gallimard, 1984, p. V-XII ; G. Saal, " "Faire voir par l'histoire” dans les mazarinades. Usages du passé entre rhétorique et bagages culturels ", dans St. Haffemayer, P. Rebollar et Y. Sordet (dir.), Mazarinades : nouvelles approches, Genève, Droz, 2016, p. 283.

16 Declaration de Messieurs les habitans de la ville de Thoulouse, op. cit., p. 6. 
Outre ces exempla constitués d'événements, les théoriciens de la rhétorique ont forgé un modèle fondé sur les personnes ${ }^{17}$. À cause du manque de moyens et de la nécessité de publier le plus rapidement possible, les libellistes ont plus régulièrement opté pour ce second type de narration, réduisant les exempla à leur portion congrue : souvent lapidaires, ils s'éloignent du modèle du petit récit pour se rapprocher de celui de la figure. Ainsi, lorsque le duc de Guise est assassiné par Henri III, Les Regrets de Madame de Nemours appellent à l'insurrection contre le "second Neron ${ }^{18}$ ». Pour comparer le roi à l'empereur romain Héliogabale, Le Martire des deux freres gagne encore en efficacité grâce à la formule « Henri Heliogabalisé ${ }^{19} »$.

Une telle décomposition du passé en figures s'inscrit dans la suite logique des préfigurations médiévales ${ }^{20}$, de l'imitatio renaissante, des recueils de vies illustres du XVI siècle $^{21}$ et des parallèles des siècles suivants ${ }^{22}$. Outre ses vertus heuristiques et pédagogiques, cette rhétorique binaire, fondée sur une conception de l'histoire comme répertoire d'exemples et de contre-exemples, permet aux polémistes d'établir des analogies propres à faire de leurs discours des « réquisitoires implacables ${ }^{23}$ ».

Tout en étant incluse dans une pratique généralisée de décomposition du passé en exempla, l'histoire romaine se démarque des autres répertoires historiques : elle est certes convoquée, à une échelle réduite, sous forme d'anecdotes et de figures, mais la société romaine tout entière est également élevée au rang d'exemple. Ainsi, la réponse aux Memoires semez par quelques politics loue la ténacité des Romains en contexte guerrier :

Si aussi bien que les Romains tu te vantes estre descendu des Troyens, cederas tu à leur vertu, toi dis[-]ie, qui as borné tes conqueste à leur Capitole. Ils perdirent contre Hannibal tout leur Empire plutost, que la moindre flammeche de leur courage [...] : ils refuserent la paix à ce Roy d'Albanie, qui la leur demandoit apres qu'il leur eust gaigné trois batailles. ${ }^{24}$

17 M. Kempshall, Rhetoric and the Writing of History [...], op. cit., p. 315-316.

18 Les Regrets de Madame de Nemours sur la mort de Messeigneurs de Guyse, ses enfants, L.O.T.H., [Paris], H. Velut, 1589, p. 7.

19 Le Martire des deux freres. Contenant au vray toutes les particularitez plus notables des Massacres, et assassinats [...], s.l., s.n., 1589, p. 17.

20 R. Koselleck, Le Futur passé. Contribution à la sémantique des temps historiques, Paris, Éd. de l'EHESS, 1999, p. 40 ; D. R. Woolf, «From Hystories to the Historical : Five Transitions in Thinking about the Past, 1500-1700 », Huntington Library Quarterly, n 68, 2005, p. 43-47.

21 Voir P. Eichel-Lojkine, op. cit. On retrouve, dans ces recueils, «l'alliance de l'exemplarité et de l'anecdotique » (p. 39) également présente dans les libelles ligueurs.

22 Voir M.-A. Bernier (dir.), Parallèle des anciens et des modernes. Rhétorique, histoire et esthétique au siècle des Lumières, Québec, Presses de l’Université Laval, 2006.

23 Ibid., p. 36.

24 Memoires semez par quelques politics aux Estats, qui se tiennent en la ville de Bloys, Avec la response Catholique à iceux, Paris, s.n., 1588, p. 15-16. 
«Les anciens Romains, les plus sages politiques du monde ${ }^{25}$ », « nation encore plus puissante et plus advisee en ses loix et polices ${ }^{26}$ ", sont des modèles de vertu, de bravoure et de défense de la res publica contre ses ennemis. Au moment où l'opposition entre les catholiques et Henri III devient frontale, c'est de la lutte des Romains contre les mauvais dirigeants que les libellistes s'emparent. Leurs textes narrent alors à plusieurs reprises comment «les Empereurs de Rome estoient deposez ou tuez, s'ils se faisoient tyrans ${ }^{27}{ }^{\text {». }}$

\section{Les galeries de figures}

De nombreux libelles ne parviennent pas à expliquer le présent par un seul exemplum : souvent, la complexité de la revendication requiert la convocation de nombreuses figures issues de l'histoire romaine pour établir l'analogie. Ainsi, afin de justifier la thèse selon laquelle "il faut chasser les mauvais dirigeants ", De la différence du Roy et du Tyran énumère :

Dequoy font foy les Histoires, comme de Sardanapale, pour avoir esté luxurieux et faineant, Denis le Tyran de Siracuse, Policrates Tyran de Samos, Tarquin le superbe Roy des Romains, les Empereurs Caligula, Neron, Galba, Vitellius, Domitian, Commodus, Heliogabale, et infinis autres, qui ont esté tuez comme Tyrans [...].28

Ce procédé, que l'on peut décrire comme une galerie de figures, apparait tout d'abord comme un héritage de Cicéron, qui énumérait des exempla avant de conclure par une injonction à leur imitation ${ }^{29}$. Pratique par laquelle le libelliste démontre l'étendue de son savoir-faire oratoire et de ses connaissances historiques, la galerie rappelle aussi la dimension agonistique à l'origine du parallèle $^{30}$. Enfin, elle est proche de la conglobation, figure de style voisine de l'accumulation ou de l'énumération ${ }^{31}$ et qui peut se définir comme une «accumulation persuasive : une démonstration faite d'un entassement plutôt que fondée sur la logique et l'observation ${ }^{32}$ » ou encore comme une «figure consistant à accumuler les arguments en faveur d'une même thèse ${ }^{33}$ ».

Certaines galeries convoquent des exempla de l'Antiquité jusqu'au présent, telle la Lettre envoyee par Messieurs de la ville de Reims, débutant avec l'empereur

25 Cl. de Bauffremont, Proposition de la Noblesse de France sur l'entretenement de l'Estat et affaires de ce Royaume, Paris, M. Buffet, 1585, f. B3v ${ }^{\circ}$

26 Remontranse faicte au Roy et à la Royne Mere, par Messieurs les Cardinaux, de Bourbon, de Guyse [...], s.l., s.n., 1586, f. A3v ${ }^{\circ}$.

27 De la difference du roy et du tyran. Dedié à M.L.L.D.M., Paris, R. Thierry, 1589, p. 37.

28 Ibid., p. 36.

29 G. Achard, Pratique rhétorique et idéologie politique dans les discours "optimates " de Cicéron, Leyde, Brill, 1981, p. 483-484.

30 Gr. Gicquiaud, «La balance de Clio : réflexions sur la poétique et la rhétorique du parallèle », dans M.-A. Bernier (dir.), Parallèle des anciens et des modernes, op. cit., p. 34-35.

31 P. Fontanier, Les Figures du discours, Paris, Flammarion, 1977, p. 363.

32 Ibid., p. 90.

33 O. Reboul, Introduction à la rhétorique : théorie et pratique, Paris, Puf, 2013, p. 237. 
Constance et s'achevant avec Elizabeth Ire, en passant par la croisade contre les Albigeois et le roi Georges de Bohême ${ }^{34}$. Dans ce cas, la galerie permet aux libellistes de souligner que la vérité qu'ils énoncent est vraie de toute éternité et, partant, d'accuser implicitement quiconque la contesterait de rompre avec un ordre millénaire, grief particulièrement incriminant durant la première Modernité35.

La Responce faicte à la declaration de Henry de Valois comporte une galerie qui, tant par la thèse qu'elle entend prouver ${ }^{36}$ que par les exempla sollicités, montre que les formes de l'anecdote et de la figure ne sont pas toujours indépendantes :

Vrayment il me souvient d'un tres grand nombre de Roys, qui ont esté engendre[z] de petis compagnons roturiers, et fort esloignez de Noblesse, toutesfois en leur Royalle dignité il se sont comportez plus modestement: entre lesquels je produiray Tarquinius Priscus, lequel fut esleu Roy des Romains, et estoit fils d'un pauvre marchant de Torinte, banni et exilé de son pays. Antipater comme recité Senecque, successeur d'Alexandre au Royaume de Macedone, estoit fils d'un bateleur. Daire, Roy des Perses, fils d'un chartier $[\ldots] .{ }^{37}$

À l'instar de la volonté revendiquée par un autre libelle de «prouver par exemples $^{38} »$, l'utilisation du terme juridique produire - désignant l'action de porter une pièce dans le débat ${ }^{39}$ - illustre comment fonctionne l'instrumentalisation de l'histoire romaine par les polémistes: les libelles, fortement influencés par le modèle de l'argumentaire judiciaire, appellent le public à émettre un jugement fondé sur la raison et sur les preuves, créant ainsi un tribunal de la lecture ${ }^{40}$. Dans ce cadre, la galerie de figures vise à créer le plus grand faisceau de convergence possible pour attester la culpabilité de celui que les catholiques accusent.

34 Lettre envoyee par Messieurs de la ville de Reims, a Nosseigneurs les deputez des Estats de France assemblez en la ville de Paris pour l'eslection d'vn Roy Catholique, Paris, R. Thierry, 1593, p. 7-11. L'auteur remercie la Bibliothèque Sainte-Geneviève pour la numérisation, à titre gracieux, de ce libelle.

35 Fr. Hartog, Régimes d'historicité. Présentisme et expérience du temps, Paris, Éd. du Seuil, 2003, p. 11 30.

36 La thèse de l'indépendance de la vertu individuelle et de celle de la naissance, voire de la supériorité de celle-là sur celle-ci, trouve son origine dans les recueils humanistes de vies d'hommes illustres : sous la plume de Jove, Boccace et Dante, l'élévation d'hommes au métier peu prestigieux grâce au seul mérite individuel devient un véritable topos (P. Eichel-Lojkine, op. cit., p. 69-71).

37 Responce faicte à la declaration de Henry de Valois sur l'innocence par luy pretendue de la mort de Messeigneurs de Guyse, s.l., jouxte la coppie Imprimée par D. Binet, 1589, p. 13-14. Nous soulignons.

38 Discours en forme d'oraison funebre, sur les massacres et parricide, de Messeigneurs le Duc, et Cardinal de Guyse [...], Paris, J. Varangue, [1589], p. 5.

39 G. Cornu, Vocabulaire juridique, Paris, Puf, 1987, p. 619.

40 T. Debbagi Baranova, À coups de libelles, op. cit., p. 454-456. 
La galerie est une nouvelle trace d'une conception du passé fondée sur l'analogie ${ }^{41}$ et l'abduction ${ }^{42}$ : elle sélectionne des références historiques éparses l'Antiquité romaine n'y échappe pas - et les purge de tout détail, notamment contextuels, afin de faire de chacune d'elles un exemplum de même valeur. Le lien entre ces figures étant créé par une situation d'élocution précise, la logique qui sous-tend la galerie est celle de l'instant et non du savoir ${ }^{43}$.

Plus qu'un héritage de Cicéron et de la rhétorique médiévale, la galerie apparaît donc comme la suite logique de la décomposition du passé en figures, dont elle effectue la mise en série. Elle souligne l'influence des recueils de vies illustres, et notamment des Vrais Pourtraicts d'André Thevet, sur la médiation ligueuse de l'Antiquité : délaissant la chronologie au profit d'une conception sérielle du passé, cet ouvrage, proche d'un catalogue, adopte en effet un classement et un regroupement thématiques des figures témoignant d'un goût prononcé pour l'anecdote ${ }^{44}$. Un semblable classement des figures par rubriques, en fonction des revendications qu'elles peuvent argumenter, se retrouve dans les recueils de lieux communs ${ }^{45}$, outils manuscrits rassemblant notamment des exempla historiques que les ecclésiastiques et les juristes, les deux grandes catégories de libellistes ligueurs ${ }^{46}$, se transmettent de génération en génération ${ }^{47}$. Une telle médiation de l'histoire est à l'origine de la conception utilitaire et analogique du passé qui trouve son expression la plus claire dans la galerie de figures : celle-ci est alors la récitation, ou plutôt la production de tous les exempla répertoriés dans une rubrique, catégorie cohérente sémantiquement et propre à permettre au passé de donner des leçons morales parfois polémiques au présent.

\section{L'apparente plasticité des exempla}

Si la signification de la majorité des exempla demeure identique ou similaire au gré de leurs mentions, certaines figures peuvent revêtir deux sens distincts selon le libelle qui les convoque. L'histoire antique n'échappe pas à cette médiation si particulière. Ainsi, Auguste est à la fois loué comme l'excellent empereur qui a "disposé tout le monde [...] à une bonne paix, et concorde, pour preparer les voyes pacifiques, à la naissance de nostre Messie Sauveur ${ }^{48}$ », puis, au cœur d'une anecdote bien différente, décrié :

41 La conglobation «touche forcément» au raisonnement analogique (P. Fontanier, op. cit., p. 90).

42 K. Abiven, "L'exemplum : un modèle opératoire dans la lettre familière ? ", art. cit., p. 4.

43 C. Saal, Le Passé en France au XVII siècle. Représentations, usages et transferts des savoirs historiques, thèse de doctorat en histoire, Université de Liège, 2015-2016, p. 132.

44 P. Eichel-Lojkine, op. cit., p. 223-234 et 341-346.

45 A. Moss, Les Recueils de lieux communs. Méthode pour apprendre à penser à la Renaissance, Genève, Droz, 2002.

46 D. Pallier, Recherches sur l'imprimerie, op. cit., p. 134 et 460-465.

47 M. Houllemare, Politiques de la parole. Le parlement de Paris au XVIe siècle, Genève, Droz, 2011, p. 160-216.

48 Remerciement faict au Roy, par Monsieur l’Archevesque de Bourges [...], s.l., s.n., 1588, p. 8. 
Auguste Cesar, pour avoir contrevenu à la loy du pays, tirant par les cheveux un quidan qui l'avoit offensé, et en cela se montrant juge et partie, fut deux jours sans manger, et cuida mourir de deuil. ${ }^{49}$

L'exemplum varie en fonction du contexte ${ }^{50}$ : quand la Ligue soutient Henri III et l'encourage à une plus grande fermeté, Auguste est un prince fort ; quand elle souhaite que les États généraux reçoivent le droit de déposer un mauvais dirigeant, il faut démontrer que l'autorité du premier empereur n'était pas absolue. Tatiana Debbagi a bien identifié ce phénomène de «compromis entre

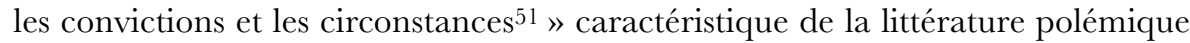
des guerres de Religion. Cette influence du contexte sur la valeur d'une figure aboutit parfois à l'inversion totale du sens de certains exempla. Ainsi, les empereurs Maximin et Dioclétien, qui faisaient partie de la galerie de roturiers au comportement noble ${ }^{52}$, sont-ils intégrés dans une liste de tyrans idolâtres ${ }^{53}$ par un autre libelle.

Préférant utiliser deux fois les mêmes figures dans des optiques opposées au lieu d'en choisir d'autres, les libellistes illustrent l'influence des recueils de lieux communs, au sein desquels les citations sont classées par rubriques, afin de permettre la défense de thèses très nombreuses et souvent incohérentes ${ }^{54}$. Selon cette médiation du passé, les diverses identités d'Auguste, de Maximin et de Dioclétien constituent chacune un exemplum à elles seules: inscrites dans des rubriques différentes, elles sont mobilisables pour prouver des thèses sans aucun rapport entre elles. Il n'y a aucune contradiction : dans la médiation rhétorique du passé, seul l'usage compte, et l'orateur sélectionne la figure la plus apte à la situation d'élocution, sans réflexion sur le contenu historique ${ }^{55}$.

\section{Girculation du savoir historique : faux-semblants et vrais recopiages}

Les libelles constituent des médiations de l'histoire romaine à part entière, mais d'autres médiations les précèdent: quelles sont les «contraintes documentaires ${ }^{56} »$ de leurs auteurs? Peut-on établir la généalogie des exempla convoqués dans les libelles ligueurs?

49 Responce faicte à la declaration de Henry de Valois, op. cit., p. 10.

50 G. Saal, " "Faire voir par l'histoire" dans les mazarinades », art. cit., p. 281.

51 T. Debbagi Baranova, À coups de libelles, op. cit., p. 32.

52 Responce faicte à la declaration de Henry de Valois, op. cit., p. 14.

53 Remonstrance tres-docte envoyee aux Catholiques François, par un Catholique Anglois, Paris, A. du Brüeil, 1589 , p. 8.

54 A. Moss, op. cit., p. 7-8.

55 M. Kempshall, op. cit., p. 265-349.

56 Fr. Mora-Lebrun et A. Rochebouet, «Introduction » à É. Anheim, P. Chastang, Fr. MoraLebrun et A. Rochebouet (dir.), L'Écriture de l'histoire au Moyen Âge. Contraintes génériques, contraintes documentaires, Paris, Classiques Garnier, 2015, p. 7-10. 
Les références que les libellistes citent parfois dans le corps ou les marges du texte renvoient presque systématiquement à des sources très prestigieuses, comme la Bible, les Pères de l'Église, ou encore les auteurs antiques - ce qui confère à l'Antiquité, romaine ou chrétienne, une omniprésence apparente. À l'instar des chroniques médiévales ${ }^{57}$, les polémistes évoquent également, comme sources de leur savoir historique, les histoires, sans plus de précision ${ }^{58}$. Ces deux types de citations de sources éclairent davantage la mise en scène de la médiation du savoir que les médiations elles-mêmes : comme les chroniqueurs médiévaux, les libellistes référencent des sources prestigieuses - ou, à défaut, restent volontairement vagues - car elles seules constituent un argument d'autorité59. Pierre Courroux a démontré qu'outre les «appels rhétoriques à la confiance $^{60}$ » qu'elles constituent, ces références «sont présentes comme un simple trompe-l'œil ${ }^{61} »$ : elles ont pour fonction de dissimuler l'utilisation de sources récentes, vernaculaires et, partant, moins prestigieuses ${ }^{62}$.

Il faut dès lors chercher les sources réellement consultées par les libellistes ailleurs que dans les références qu'ils revendiquent. Si les recueils de lieux communs représentent assurément la médiation la plus fréquente, des recopiages depuis des sources de première main peuvent aussi être identifiés grâce à des recherches intertextuelles. Plus l'exemplum est précis et anecdotique, plus l'identification de son origine est aisée. Ainsi, la désormais fameuse galerie de rois roturiers au noble comportement trouve son origine dans L'Histoire de Chelidonius Tigurinus, publiée à Paris en 1556 par l'écrivain et compilateur français Pierre Boaistuau63. Le recopiage est presque intégral: l'ordre dans lequel les exempla sont disposés est identique, les deux textes utilisent les mêmes expressions et citent la même source prestigieuse, Sénèque ${ }^{64}$, pur écran de fumée. Les citations de paroles sont également des informations dont l'origine est aisément décelable, telles celles de l'empereur Antonin que l'Avis à l'irresolu de

57 P. Courroux, " "Si com jou truis el livre escrit". Les références externes dans la Chronique rimée de Philippe Mousket », ibid., p. 167-168.

58 «Messieurs, les histoires tesmoignent que les Empereurs, et Rois Chrestiens... » (Memoires semez par quelques politics aux Estats, op. cit., p. 3). Parfois, une précision très générale est fournie : certains libelles se réfèrent aux «Histoires, tant sainctes que prophanes » (Le Martyre de Frere facques Clement de l'ordre S. Dominique, Troyes, J. Moreau, [1589], p. 6), à « l'Histoire de France » (Discours veritable de ce qui est advenu aulx Estats generaulx de France tenuz a Bloys en l'annee 1588, s.l., s.n., 1589, p. 35), etc.

59 P. Courroux, " "Si com jou truis el livre escrit" », art. cit., p. 161-166.

60 P. Courroux, L'Écriture de l'histoire, op. cit., p. 172.

61 P. Courroux, art. cit., p. 162.

62 Voir P. Courroux, L'Écriture de l'histoire, op. cit., p. 453-472 ; P. Burke, The Renaissance Sense of the Past, Londres, R. Cunningham, 1969, p. 1-7.

63 P. Boaistuau, L'Histoire de Chelidonius Tigurinus [...], Paris, V. Sertenas, 1556, f. $123 \mathrm{r}^{\circ}-\mathrm{v}^{\circ}$.

64 «Comme Seneque nous tesmoigne» (ibid., f. 123v $\mathrm{v}^{\circ}$; «Comme recite Senecque » (Responce faicte à la declaration de Henry de Valois, op. cit., p. 13). 
Limoges $^{65}$ recopie des Mémoires de l'estat de France 66 publiés en 1576 par Simon Goulart.

Les véritables sources des libellistes sont donc ces recueils historiques que le XVI ${ }^{\mathrm{e}}$ siècle imprima par dizaines ${ }^{67}$. Ces grandes compilations de manuscrits antiques et médiévaux sont des outils très précieux pour les polémistes cherchant à convoquer le plus grand nombre d'exempla mais, composées en langue vernaculaire moins d'un siècle avant la Ligue, elles ne possèdent pas une autorité suffisante pour que leur titre soit cité, bien que les libellistes en reproduisent de longs extraits. Ce procédé n'est nullement propre aux libelles catholiques. Tatiana Debbagi a bien identifié cette «accommodation des textes» caractéristique de la culture politique des guerres de Religion : pour être le premier à réagir, on fait feu de tout bois ; dans cette optique, le recopiage est un procédé très commode ${ }^{68}$. Les polémistes de la Ligue y ont recours à de très nombreuses reprises ${ }^{69}$, et n'hésitent pas à recopier des auteurs protestants : Goulart est un polémiste huguenot réfugié à Genève, et ses Memoires de l'Estat de France sont un véritable réquisitoire anticatholique ${ }^{70}$. Cette migration des textes d'un camp à l'autre est représentative des pratiques polémiques des guerres de Religion ${ }^{71}$.

En sondant les sources d'inspiration des libelles, le chercheur découvre parfois des textes postérieurs mentionnant des passages identiques. Ainsi, on retrouve la galerie des rois roturiers au noble comportement dans les Observations et maximes ${ }^{72}$ publiées à Paris en 1715 par l'avocat Antoine Bruneau, et l'exemplum du capitaine romain Camille, issu de la Harangue prononcee devant le Roy ${ }^{73}$, dans les Eloges et vies des reynes ${ }^{74}$ du minime français Hilarion de Coste, parus à Paris en 1630. S'agit-il d'indices d'une "productivité pamphlétaire ${ }^{75} »$ ? Le libelle constitue-t-il la médiation de l'histoire romaine entre la source qu'il recopie et ces textes postérieurs, ou ces derniers se sont-ils servis de la même source que les

65 Avis à l'irresolu de Limoges. Qui peut servir à toutes les villes [...], Paris, R. Le Fizelier, 1589, p. 66.

66 S. Goulart, Memoires de l'estat de France, sous Charles neufiesme, t. III, s.l., s.d., 1576, p. 70.

67 Voir Cl.-G. Dubois, La Conception de l'histoire en France au XVIe siècle (1560-1610), Paris, Nizet, 1977 ; D. R. Woolf, «From Hystories to the Historical », art. cit.

68 Voir T. Debbagi Baranova, À coups de libelles, op. cit., p. 189-206.

69 Les Regrets et souspirs lamentables de la France sur le tres-pas de tres-haut et tres-valeureux Prince, mon Seigneur François de Valois [...], Paris, F. Tabert, 1584; éd. A. Goderniaux, Liège, Bibliothèque Alpha, XXII.87.8(R), pièce n³, Arm@rium Universitatis Leodiensis. La Bibliothèque numérique du Moyen Âge et de la première Modernité de l'Université de Liège, éd. en ligne: http://hdl.handle.net/ 2268.1/3964.

70 C. Huchard, D'encre et de sang. Simon Goulart et la Saint-Barthélemy, Paris, H. Champion, 2007.

71 T. Debbagi Baranova, op. cit., p. 189-206 ; Les Regrets et souspirs lamentables de la France, éd. cit.

72 A. Bruneau, Observations et maximes sur les matieres criminelles, Paris, G. Cavelier, 1715, p. 279-280.

73 E. Bernard, Harangue prononcee devant le Roy, seant en ses Estats generaux tenus à Bloys [...], Lyon, J. Pillehotte, 1589, p. 15.

74 H. de Coste, Les Eloges et vies des reynes, princesses, dames et damoiselles illustres [...], Paris, S. Cramoisy, 1630, p. 300.

75 L'auteur souhaite exprimer ici sa gratitude à Delphine Amstutz pour la suggestion de cette judicieuse formule. 
libellistes ? Établir le lien précis entre ces deux textes et les libelles catholiques nécessiterait une enquête approfondie de la biographie, de la bibliothèque et des écrits de Bruneau et de Coste.

\section{Conclusion : les libelles ligueurs au cœur de deux mille ans de médiations}

Les auteurs des libelles ne sont pas des historiens. Leur activité polémique n'est en rien liée aux différentes proto-méthodes historiques qui voient le jour au $\mathrm{XVI}{ }^{\mathrm{e}}$ siècle $^{76}$. Ils ont en revanche compris toutes les subtilités de l'art oratoire, et mettent leur talent rhétorique au service du parti ligueur. L'histoire antique n'échappe pas à la vaste instrumentalisation induite par la technique rhétorique de l'exemplum. Les nombreuses médiations qui en résultent sont la trace d'une très grande diversité des pratiques: les polémistes convoquent simultanément des figures et des épisodes, les disposent en exempla indépendants autant qu'en galeries, alternent stabilité et plasticité. Ces textes longtemps critiqués pour leur faible qualité littéraire ${ }^{77}$ témoignent en réalité d'une grande créativité. Leurs pratiques discursives, riches et complexes, se situent à la croisée du Moyen Âge et de l'Âge classique.

Les libellistes bâtissent cette médiation de l'histoire antique sur des médiations préalables qu'ils soumettent à un vaste processus de recopiage. Alliant servilité et inventivité, ce dernier illustre lui aussi la richesse des pratiques discursives des polémistes. On peut ainsi reconstituer un très long héritage culturel : plusieurs textes manuscrits de l'Antiquité tardive et du Moyen Âge en constituent la source écrite originelle; les grands recueils historiques les compilant aux débuts de l'ère de l'imprimerie forment un substrat commun à de nombreux lecteurs dotés des intentions les plus diverses; les recueils de lieux communs qui en extraient les exempla sont une médiation plus personnelle, souvent familiale ou amicale; les libelles marquent une étape supplémentaire, fondée sur des revendications politiques, elles-mêmes dictées par le contexte; enfin, certains textes postérieurs se fondent peut-être sur ces libelles pour utiliser les mêmes arguments issus de l'histoire romaine. Deux étapes supplémentaires pourraient être intercalées: entre les chroniques et les recueils de lieux communs, les grammaires et les manuels de rhétorique inventorient de nombreux exempla; après les textes inspirés par les libelles, les dictionnaires et les encyclopédies institutionnalisent le savoir hérité de cette longue chaîne de médiations.

76 Cl.-G. Dubois, La Conception de l'histoire en France au XVI siècle, op. cit.

77 M. Penzi, «Damnatio memoria. La Ligue Catholique française e la storiografia, tra politiques, rivoluzionari, mistici e liberali », Quaderni storici, $\mathrm{n}^{\circ} 118 / 1,2005$, p. 263-284. 


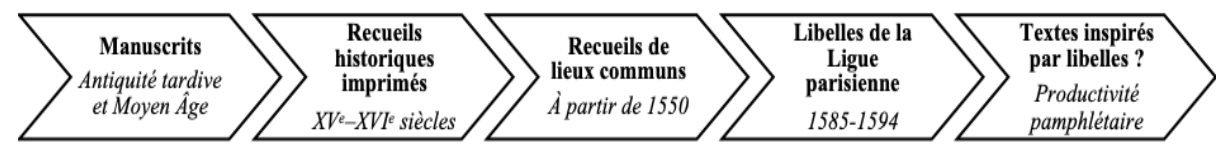

D’un point de vue méthodologique, ces résultats et la démonstration qui permet d'y aboutir ont au moins deux conséquences. Premièrement, ils confirment le rôle majeur de la rhétorique, méthode fondamentale de pensée et d'expression de toute la première Modernité. Ensuite, s'il est bien connu que convaincre est l'objectif principal, sinon unique des libellistes, les actions concrètes mises en œuvre à cette fin demeurent méconnues. Conciliant histoire et analyse du discours, notre enquête aura peut-être permis de mieux comprendre, grâce à l'analyse de pratiques concrètes, l'instrumentalisation de l'écrit durant les guerres de Religion.

Alexandre Goderniaux

Université de Liège

UR Transitions 